\title{
THE SOLUTION BY ITERATION OF NONLINEAR EQUATIONS IN HILBERT SPACES
}

\author{
ŞT. MĂRUŞTER
}

\begin{abstract}
The weak and strong convergence of the iterates generated by $x_{k+1}=\left(1-t_{k}\right) x_{k}+t_{k} T x_{k}\left(t_{k} \in R\right)$ to a fixed point of the mapping $T$ : $C \rightarrow C$ are investigated, where $C$ is a closed convex subset of a real Hilbert space. The basic assumptions are that $T$ has at least one fixed point in $C$, and that $I-T$ is demiclosed at 0 and satisfies a certain condition of monotony. Some applications are given.
\end{abstract}

Introduction. Let $H$ be a real Hilbert space, $C$ a closed convex subset of $H$ and $T$ a nonlinear mapping of $C$ into $C$ with the nonempty fixed point set $F$ in $C$. The mapping $T$ is said to be monotone if $\langle T x-T y, x-y\rangle \geqslant 0$ for all $x, y \in C$. According to [2] and [8], the mapping $T$ is said to be demiclosed at 0 in $C$ if $\left\{u_{k}\right\}$ is a sequence in $C$ which converges weakly to $u \in C$, and if $\left\{T u_{k}\right\}$ converges strongly to zero, then $T u=0$. In this paper we study the convergence of the sequence of iterates generated by

$$
x_{k+1}=\left(1-t_{k}\right) x_{k}+t_{k} T x_{k} \quad\left(x_{0} \in C\right) \text {, }
$$

where $t_{k} \in R, k=0,1, \ldots$, under the basic assumptions that $I-T$ satisfies a particular condition of monotony and that $I-T$ is demiclosed at 0 in C.

The main theorems. The mapping $T$ will be said to satisfy condition (A) if $F$ is nonempty and if there exists a real positive number $\lambda$ such that

$$
\langle x-T x, x-\xi\rangle \geqslant \lambda\|x-T x\|^{2}, \quad \forall x \in C, \xi \in F .
$$

It is obvious that (2) is a particular condition of monotony of $I-T$.

THEOREM 1. Let $T: C \rightarrow C$ be a nonlinear mapping, where $C$ is a closed convex subset of $H$. Suppose that $T$ satisfies condition (A), $I-T$ is demiclosed at 0 in $C$ and the sequence $\left\{x_{k}\right\}$ generated by (1) with $0<a \leqslant t_{k} \leqslant b<2 \lambda$ belongs to $C$. Then $\left\{x_{k}\right\}$ converges weakly to an element of $F$.

Proof. From (2) we obtain

Received by the editors March 26, 1975 and, in revised form, July 24, 1975 and September 24, 1976.

AMS (MOS) subject classifications (1970). Primary 47H10.

Key words and phrases. Iteration, fixed points, demiclosed mappings, monotone mappings.

(c) American Mathematical Society 1977 


$$
\begin{aligned}
\left\|x_{k+1}-\xi\right\|^{2} & =\left\|x_{k}-\xi-t_{k}\left(x_{k}-T x_{k}\right)\right\|^{2} \\
& =\left\|x_{k}-\xi\right\|^{2}-2 t_{k}\left\langle x_{k}-T x_{k}, x_{k}-\xi\right\rangle+t_{k}^{2}\left\|x_{k}-T x_{k}\right\|^{2} \\
& \leqslant\left\|x_{k}-\xi\right\|^{2}-t_{k}\left(2 \lambda-t_{k}\right)\left\|x_{k}-T x_{k}\right\|^{2} .
\end{aligned}
$$

Since $2 \lambda-t_{k}>0$, it follows that $\left\|x_{k+1}-\xi\right\| \leqslant\left\|x_{k}-\xi\right\|$ and so $\left\|x_{k}-\xi\right\| \rightarrow$ $\rho_{\xi}$ as $k \rightarrow \infty$ for all $\xi \in F$. From $0<a \leqslant t_{k} \leqslant b<2 \lambda$ and from the above relation, it follows that

$$
\left\|x_{k}-T x_{k}\right\|^{2} \leqslant(a(2 \lambda-b))^{-1}\left(\left\|x_{k}-\xi\right\|^{2}-\left\|x_{k+1}-\xi\right\|^{2}\right) \rightarrow 0 \quad(k \rightarrow \infty) .
$$

Since the sequence $\left\{x_{k}\right\}$ is bounded, there exists a subsequence $\left\{x_{k_{j}}\right\}$ of $\left\{x_{k}\right\}$ which converges weakly to an $x^{*}$; since $\left\{x_{k_{j}}\right\} \subset C$ and $C$ is closed and convex (hence weakly closed), it follows that $x^{*} \in C$. Moreover, $x^{*}$ is a fixed point of $T$, since $x_{k_{j}}-T x_{k_{j}} \rightarrow 0$ and $I-T$ is demiclosed at 0 (hence $x^{*}-$ $T x^{*}=0$ ).

Suppose there are two subsequences of $\left\{x_{k}\right\}$, say $\left\{u_{k}\right\}$ and $\left\{v_{k}\right\}$, which converge weakly to $u$ and $v$, respectively. As above, we have that $u$ and $v$ are in $F$ and, hence,

$$
\left\|x_{k}-u\right\| \rightarrow \rho_{u}, \quad\left\|x_{k}-v\right\| \rightarrow \rho_{v} .
$$

Now, consider the sequence

$$
E_{k}=\left\|u_{k}-u\right\|^{2}-\left\|v_{k}-u\right\|^{2}-\left\|u_{k}-v\right\|^{2}+\left\|v_{k}-v\right\|^{2} .
$$

Since relations (3) hold for any subsequence of $\left\{x_{k}\right\}$ (in particular, for $\left\{u_{k}\right\}$ and $\left.\left\{v_{k}\right\}\right)$, it follows that $E_{k} \rightarrow 0$ as $k \rightarrow \infty$. On the other hand, by a simple computation, we have

$$
E_{k}=-2\left\langle u_{k}-v_{k}, u-v\right\rangle .
$$

This and the weak convergence of $\left\{u_{k}\right\}$ and $\left\{v_{k}\right\}$ to $u$ and $v$, respectively, imply that $E_{k} \rightarrow-2\|u-v\|^{2}$ and, hence, $\|u-v\|=0$, i.e., $u=v$. Therefore, all weakly convergent subsequences of $\left\{x_{k}\right\}$ have the same weak limit, say $x^{*}$. It follows that $x_{k} \rightarrow x^{*}$ as $k \rightarrow \infty$ and the theorem is proved.

REMARK. If $T$ is quasi-nonexpansive (i.e. $\|T x-\xi\| \leqslant\|x-\xi\|$ for all $x \in C$ and $\xi \in F$ ) then (2) is satisfied with $\lambda=\frac{1}{2}$. In this case we obtain a result of W. G. Dotson [4], which is, in turn, a generalization of a theorem of H. Schaefer [9].

A similar condition of monotony was considered by J. B. Diaz and F. T. Metcalf [3], namely (with our notation): there exists $\lambda>0$ such that $\langle x-$ $T x, x-\xi\rangle>(\lambda / 2)\|x-T x\|^{2}$ for all $x \in C$ and $\xi \in F$. The above-mentioned authors have proved that if $F$ is nonempty and $T$ is a continuous mapping, then the sequence $\left\{x_{k}\right\}$ given by (1) with $t_{k}=\lambda$ either contains no strongly convergent subsequence or $\left\{x_{k}\right\}$ is strongly convergent to an element of $F$. A similar result can be obtained under the weaker assumption that $I-T$ is demiclosed at 0 ( $T$ is not necessarily continuous).

From the point of view of applications it is interesting to obtain additional conditions such that the sequence $\left\{x_{k}\right\}$ converges strongly to an element of $F$. 
In a recent paper, [10] H. F. Senter and W. G. Dotson considered the following condition: There is a nondecreasing function $f:[0, \infty) \rightarrow[0, \infty)$ with $f(0)=0$ and $f(r)>0$ for $r>0$, such that $\|x-T x\| \geqslant f(d(x, F))$ for all $x \in C$, where $d(x, F)=\inf \{\|x-z\|: z \in F\}$. In [7], C. Outlaw considered the more restrictive case, $\|x-T x\| \geqslant c \cdot \sup \{\|x-z\|: z \in F\}$.

TheOREM 2. Let $T$ be as in Theorem 1. If, in addition, there is $h \in C, h \neq 0$, such that $\langle x-T x, h\rangle \leqslant 0$ for all $x \in C$, then the sequence $\left\{x_{k}\right\}$ generated by (1) with $0<a \leqslant t_{k} \leqslant b<2 \lambda$ and for suitable $x_{0}$ in $C$, converges strongly to an element of $F$.

Proof. By Theorem 1, it follows that $x_{k} \rightarrow x^{*} \in F$ as $k \rightarrow \infty$. Suppose that $\left.\left\langle x_{0}, h\right\rangle\right\rangle\left\langle x^{*}, h\right\rangle$; then there exists $\varepsilon>0$ such that

$$
\left\langle x_{0}-x^{*}, h\right\rangle \geqslant \varepsilon\left\|x_{0}-x^{*}\right\|^{2} \text {. }
$$

If we suppose that

$$
\left\langle x_{k}-x^{*}, h\right\rangle \geqslant \varepsilon\left\|x_{k}-x^{*}\right\|^{2},
$$

then from (2) and from the fact that $\langle x-T x, h\rangle \leqslant 0$, it follows that $\left\langle x_{k+1}-x^{*}, h\right\rangle \geqslant \varepsilon\left\|x_{k+1}-x^{*}\right\|^{2}$, that is, (5) holds for every $k$. Since $x_{k} \rightarrow$ $x^{*}$ as $k \rightarrow \infty$, it follows that $\left\|x_{k}-x^{*}\right\| \rightarrow 0$, which proves the theorem.

Linear equations. As an application of Theorem 2, we obtain the convergence of some iteration scheme for linear equations in Hilbert spaces, without the assumption of compactedness of the mapping. We have

Theorem 3. Let $B: H \rightarrow H$ be a continuous linear mapping and $f \in B(H)$. Suppose zero is an eigenvalue of the mapping $B$ and the following condition is satisfied.

$$
\langle B y, y\rangle \geqslant \lambda\|B y\|^{2}, \quad \forall y \in H,
$$

where $\lambda>0$. Then the sequence $\left\{x_{k}\right\}$, generated by $x_{k+1}=x_{k}-t_{k}\left(B x_{k}-f\right)$ with $0<a \leqslant t_{k} \leqslant b<2 \lambda$ and for suitable $x_{0}$ in $H$, converges strongly to $a$ solution of the equation $B x-f=0$.

Proof. Apply Theorem 2 with $C=H$ and $T x=x-B x+f$. Since $f \in$ $B(H)$, there exists $\xi \in H$ such that $B \xi-f=0$ and, hence, $x-T x=$ $B(x-\xi)$. It is easy to see that condition (A) is satisfied (indeed, $F \neq \varnothing$ and if in (6) we put $y=x-\xi$ we obtain (2)). Suppose $x_{k} \rightarrow x^{*}$ and $B x_{k}-f \rightarrow 0$ as $k \rightarrow \infty$; then $\left\|B x^{*}-f\right\|^{2}=\lim \left\langle B x_{k}-f, B x^{*}-f\right\rangle=0$ and, hence, $B x^{*}$ $-f=0$. Therefore, $I-T$ is demiclosed at 0 . Finally, since zero is an eigenvalue of $B$ (hence also of the adjoint $B^{*}$ of $B$ ), it follows that there exists $h \neq 0$ such that $B^{*} h=0$. Therefore, $\langle B(x-\xi), h\rangle=\left\langle x-\xi, B^{*} h\right\rangle=0$ for all $x \in H$ and the theorem is proved.

Remark. J. B. Diaz and F. T. Metcalf [3] proved that if $B$ is compact, semipositive (i.e., $\langle B x, x\rangle \geqslant 0$ for all $x \in H$ ) and selfadjoint, then (6) is satisfied with $\lambda=1 / \lambda_{1}$, where $\lambda_{1}$ is the largest eigenvalue of $B$. 
The relaxation method for linear inequalities. This method, given by $\mathrm{S}$. Agmon [1], T. S. Motzkin and I. I. Schoenberg [6], is a nontrivial example for Theorem 1 in the finite dimensional case.

Let $E_{n}$ be the real $n$-dimensional Euclidean space and let $M_{i} \subset E_{n}$ ( $i=$ $1, \ldots, m)$ be a family of closed convex subsets of $E_{n}$ with nonempty intersection, $\cap M_{i} \neq \varnothing$. We are interested in determining an element of $\cap M_{i}$.

Let $x \in E_{n}$ and $\pi(x, i)$ be the projection of $x$ onto $M_{i}$ (if $x \in M_{i}$, then $\pi(x, i)=x)$. Let $i_{x}$ be the least index such that

$$
\left\|x-\pi\left(x, i_{x}\right)\right\|=\max _{i}\|x-\pi(x, i)\| .
$$

We define the mapping $T: E_{n} \rightarrow E_{n}$ by $T x=\pi\left(x, i_{x}\right)$. It is clear that $x \in$ $\cap M_{i}$ if and only if $T x=x$, hence if and only if $x$ is a fixed point of $T$.

THEOREM 4. The sequence $\left\{x_{k}\right\}$ generated by

$$
x_{k+1}=\left(1-t_{k}\right) x_{k}+t_{k} \pi\left(x_{k}, i_{x_{k}}\right) \quad\left(x_{0} \in E_{n}\right),
$$

with $0<a \leqslant t_{k} \leqslant b<2$, converges to an element of $\cap M_{i}$.

Proof. Apply Theorem 1 with $C=E_{n}$ and $T x=\pi\left(x, i_{x}\right)$. From the above remark, $F=\cap M_{i} \neq \varnothing$. Let $x \in E_{n}$ and $\xi \in F$. Since $\pi\left(x, i_{x}\right)$ is the projection of $x$ onto $M_{i_{x}}$ and $\xi \in M_{i_{x}}$, it follows that $\left\langle x-\pi\left(x, i_{x}\right), \pi\left(x, i_{x}\right)-\xi\right\rangle \geqslant$ 0 . Therefore we have

$$
\begin{aligned}
& \langle x-T x, x-\xi\rangle=\left\langle x-\pi\left(x, i_{x}\right), x-\xi\right\rangle \\
& \quad=\left\langle x-\pi\left(x, i_{x}\right), x-\pi\left(x, i_{x}\right)\right\rangle+\left\langle x-\pi\left(x, i_{x}\right), \pi\left(x, i_{x}\right)-\xi\right\rangle \\
& \quad \geqslant\left\|x-\pi\left(x, i_{x}\right)\right\|^{2}=\|x-T x\|^{2},
\end{aligned}
$$

hence we obtain relation (2) with $\lambda=1$.

It remains to show that $I-T$ is demiclosed at 0 . Let $\left\{x_{k}\right\} \subset E_{n}$ be such that $x_{k} \rightarrow x^{*}$ and $x_{k}-T x_{k} \rightarrow 0$ as $k \rightarrow \infty$. For each $i(1 \leqslant i \leqslant m)$ we have

$$
\left\|x_{k}-\pi\left(x_{k}, i\right)\right\| \leqslant\left\|x_{k}-\pi\left(x_{k}, i_{x_{k}}\right)\right\|=\left\|x_{k}-T x_{k}\right\| \rightarrow 0, \quad k \rightarrow \infty .
$$

Since $\pi(x, i)$ is a continuous function for each $i$ and $E_{n}$ is a finite dimensional space (hence $\left\{x_{k}\right\}$ converges strongly to $x^{*}$ ), it follows that

$$
\lim _{k \rightarrow \infty}\left\|x_{k}-\pi\left(x_{k}, i\right)\right\|=\left\|x^{*}-\pi\left(x^{*}, i\right)\right\|=0
$$

for each $i(1 \leqslant i \leqslant m)$. Therefore, $x^{*}-T x^{*}=x^{*}-\pi\left(x^{*}, i_{x^{*}}\right)=0$ and so $I-T$ is demiclosed at 0 .

From Theorem 1, the sequence $\left\{x_{k}\right\}$ generated by (7) converges weakly (and thus strongly) to an element of $F=\cap M_{i}$. This proves Theorem 4.

Remarks. It is easy to see that $T x=\pi\left(x, i_{x}\right)$ is a discontinuous function in those points $x \in E_{n}$ where $\max _{i}\|x-\pi(x, i)\|$ is touched for more than one value of index $i$.

Theorem 4 is due to I. I. Eremin [5]. Our method of proof seems to be simpler. 
Theorem 4 contains, as a special case, a result obtained by S. Agmon [1], T. S. Motzkin and I. I. Schoenberg [6] for the case when $M_{i}$ is defined by the inequalities of the form

$$
\sum_{j=1}^{n} a_{i j} x_{j}+b_{i} \geqslant 0, \quad i=1, \ldots, m
$$

\section{REFERENCES}

1. S. Agmon, The relaxation method for linear inequalities, Canad. J. Math. 6 (1954), 382-392. MR 16, 18.

2. F. E. Browder and W. V. Petryshyn, The solution by iteration of nonlinear functional equations in Banach spaces, Bull. Amer. Math. Soc. 72 (1966), 571-575. MR 32 \#8155b.

3. J. B. Diaz and F. T. Metcalf, On the set of subsequential limit points of successive approximations, Trans. Amer. Math. Soc. 135 (1969), 459-485. MR 38 \#2644.

4. W. G. Dotson, Jr., On the Mann iterative process, Trans. Amer. Math. Soc. 149 (1970), 65-73. MR 41 \#2477.

5. I. I. Eremin, The generalization of the relaxation method of Motzkin and Agmon, Uspehi Mat. Nauk 20 (1965), no. 2 (122), 183-187. (Russian) MR 34 \#2342.

6. T. S. Motzkin and I. J. Schoenberg, The relaxation method for linear inequalities, Canad. J. Math. 6 (1954), 393-404. MR 16, 18.

7. C. Outlaw, Mean value iteration of nonexpansive mappings in a Banach space, Pacific $J$. Math. 30 (1969), 747-750. MR 40 \#807.

8. W. V. Petryshyn and T. E. Williamson, Jr., Strong and weak convergence of the sequence of successive approximations for quasi-nonexpansive mappings, J. Math. Anal. Appl. 43 (1973), 459-497. MR 48 \# 4854.

9. H. Schaefer, Über die Methode sukzessiver Approximationen, Jber Deutsch. Math. Verein. 59 (1957), Abt. 1, 131-140. MR 18, 811.

10. H. F. Senter and W. G. Dotson, Approximating fixed points of nonexpansive mappings, Proc. Amer. Math. Soc. 44 (1974), 375-380. MR 49 \#11333

Department of Mathematics, University of Timisoara, B. Pírvan 4, Timişoara, Romania 